\title{
New physics effects on neutrinoless double beta decay from right-handed current
}

\author{
Shao-Feng Ge, ${ }^{a}$ Manfred Lindner ${ }^{a}$ and Sudhanwa Patra ${ }^{a, b}$ \\ ${ }^{a}$ Max-Planck-Institut für Kernphysik, \\ Saupfercheckweg 1, Heidelberg, 69117 Germany \\ ${ }^{b}$ Centre of Excellence in Theoretical and Mathematical Sciences, Siksha O Anusandhan Univeristy, \\ Khandagiri Square, Bhubaneswar, Odisha, 751030 India \\ E-mail: gesf02@gmail.com, lindner@mpi-hd.mpg.de, \\ sudhanwa@mpi-hd.mpg.de
}

ABSTRACT: We study the impact of new physics contributions to neutrinoless double beta decay arising from right-handed current in comparison with the standard mechanism. If the light neutrinos obtain their masses from Type-II seesaw within left-right symmetric model, where the Type-I contribution is suppressed to negligible extent, the right-handed PMNS matrix is the same as its left-handed counterpart, making it highly predictable and testable at next-generation experiments. It is very attractive, especially with recent cosmological constraint favoring the normal hierarchy under which the neutrinoless double beta decay is too small to be observed unless new physics appears as indicated by the recent diboson excess observed at ATLAS. The relative contributions from left- and right-handed currents can be reconstructed with the ratio between lifetimes of two different isotopes as well as the ratio of nuclear matrix elements. In this way, the theoretical uncertainties in the calculation of nuclear matrix elements can be essentially avoided. We also discuss the interplay of neutrinoless double beta decay measurements with cosmology, beta decay, and neutrino oscillation.

Keywords: Rare Decays, Beyond Standard Model, Neutrino Physics

ARXIV EPRINT: 1508.07286 


\section{Contents}

1 Introduction $\quad 1$

2 Neutrinoless double beta decay 2

3 Effect of new physics contribution 5

$\begin{array}{lll}3.1 & \text { Type-II seesaw dominance } & 6\end{array}$

4 Comparison between new physics and standard mechanism $\quad 7$

5 Separating the SM and LRSM contributions 11

6 Conclusions 13

A Maximum and minimum values for effective Majorana mass parameter 13

\section{Introduction}

Although the Standard Model (SM) of particle physics is extremely successful in explaining most of the experimental data up to current accelerator energies, it does not explain nonzero neutrino masses confirmed by solar, atmospheric and reactor oscillation data, as well as the mystery of dark matter and the matter dominance of present universe. These are the experimentally facts which motivate physics beyond the standard model and in addition there exist various theoretical reasons.

Left-Right Symmetric Models (LRSM) [1-6] are well motivated candidates of physics beyond SM. Among the reasons are: i) explaining light neutrino masses via seesaw mechanism with the natural embedding of right-handed neutrinos, ii) providing theoretical origin of maximal parity violation seen at weak interaction while conserved in strong and electromagnetic interaction, iii) the fact that $B-L$ is a more attractive $\mathrm{U}(1)$ quantum number and the symmetry of $\mathrm{U}(1)$ charges of the fermions. With the scalar sector comprising of Higgs bidoublet and triplets, the light neutrino mass is generated by type-I plus type-II seesaw mechanism. The Higgs scalars generate masses for light and heavy neutrinos which not only accommodates the Majorana nature of light and heavy neutrinos, but also leads to testable lepton number violation (LNV) at low energy experiments like neutrinoless double beta decay $(0 \nu \beta \beta)$ and at high energy experiments like the Large Hadron Collider (LHC, see ref. [7] for a detailed discussions). The LHC can also probe right-handed current. Recently ATLAS [8] claims to find a diboson excess which can be explained by a $2 \mathrm{TeV}$ $W^{\prime}$ via $p p \rightarrow W^{\prime} \rightarrow W Z$, and CMS [9-11] sets stringent lower bound on the $W^{\prime}$ mass through $p p \rightarrow W^{\prime} \rightarrow \ell \ell+2 j$. In the context of LRSM, in principle, one can derive limits 
for right-handed neutrino mass and mixing, $W_{\mathrm{R}}$ mass and its mixing with the left-handed counterpart as well as the corresponding gauge coupling $g_{\mathrm{R}}$.

On the other hand, neutrinoless double beta decay is a unique phenomena whose experimental observation would reveal the character of light neutrinos i.e, whether they have Majorana mass term [12] which violates lepton number. If so, neutrinoless double beta decay can happen. At present, the best lower limit on the decay half-life using ${ }^{76} \mathrm{Ge}$ is $T_{1 / 2}^{0 \nu}>2.1 \times 10^{25} \mathrm{yrs}$ at $90 \%$ C.L. from GERDA [13] while the combined bound is $T_{1 / 2}^{0 \nu}>3.0 \times 10^{25}$ yrs. At the same time, the future experiment GERDA Phase-II [14] is expected to improve the half-life sensitivity to reach $T_{1 / 2}^{0 \nu}>2.0 \times 10^{26}$ yrs. For ${ }^{136} \mathrm{Xe}$, the derived lower limits on half-life from EXO-200 and KamLAND-Zen experiments are $T_{1 / 2}^{0 \nu}>1.6 \times 10^{25}$ yrs [15] and $T_{1 / 2}^{0 \nu}>1.9 \times 10^{26}$ yrs [16], respectively. The combined limit from KamLAND-Zen collaboration is $T_{1 / 2}^{0 \nu}>3.4 \times 10^{26}$ yrs at $90 \%$ C.L.

Lepton number violating $0 \nu \beta \beta$ transition could be induced by either light Majorana neutrinos or new physics. For the latter, one has to go beyond SM and many models can contribute to neutrinoless double beta decay [7, 17-30]. The nest-generation experiments of neutrinoless double decay can touch down to the lower limit of inverted hierarchy (IH), corresponding to effective Majorana mass parameter $m_{e e}^{\nu}$ around $0.01 \mathrm{eV}$. Nevertheless, the latest cosmological bound on the mass sum of light active neutrinos [32] has indicated that the normal hierarchy $(\mathrm{NH})$ is favored at $1 \sigma$ confidence level with tiny smallest mass. A direct consequence is that neutrinoless double beta decay is difficult to be observed at next-generation experiments if its only source comes from the standard mechanism. If the result of observation turns out to be the opposite, namely neutrinoless double beta decay is observed but cannot be explained by the standard mechanism, it clearly indicates physics beyond SM.

With these motivations, both hints from the diboson excess observed at ATLAS for heavy $W^{\prime}$ around $2 \mathrm{TeV}$ and cosmological preference for $\mathrm{NH}$ with tiny mass scale and invisible neutrinoless double beta decay even at next-generation experiments, we examine the effect of Type-II seesaw contributions to neutrinoless double beta decay arising from purely right-handed current within LRSM. The predicted effect mass can saturate the current experimental bounds making it testable in the future even with $\mathrm{NH}$ being favored.

The paper is organized as follows: after briefly summarizing the standard mechanism of neutrinoless double beta decay in section 2 we consider new physics effect from purely right-handed current in section 3. Next we compare the new physics contribution with the standard mechanism and derive lower limit on the absolute scale of light neutrinos from current experimental limit in section 4. In section 5, we use half-life and nuclear matrix element (NME) ratios to reconstruct the relative contributions of the standard mechanism and new physics to effective Majorana mass parameter. This approach to distinguishing different sources has a significant advantage that the theoretical uncertainties in the calculation of NME can be essentially avoided. We conclude our paper in section 6 .

\section{Neutrinoless double beta decay}

Observing neutrinoless double beta decay can not only reveal LNV but also provide crucial information about the absolute scale of light neutrino masses and the associated mass- 


\begin{tabular}{|cc|}
\hline Oscillation Parameters & $3 \sigma$ range \\
\hline$\Delta m_{\mathrm{s}}^{2}\left[10^{-5} \mathrm{eV}^{2}\right]$ & $7.11-8.18$ \\
$\left|\Delta m_{\mathrm{a}}^{2}\right|\left[10^{-3} \mathrm{eV}^{2}\right](\mathrm{NH})$ & $2.30-2.65$ \\
$\left|\Delta m_{\mathrm{a}}^{2}\right|\left[10^{-3} \mathrm{eV}^{2}\right](\mathrm{IH})$ & $2.20-2.54$ \\
\hline $\sin ^{2} \theta_{\mathrm{s}}$ & $0.278-0.375$ \\
$\sin ^{2} \theta_{\mathrm{r}}(\mathrm{NH})$ & $0.0177-0.0294$ \\
$\sin ^{2} \theta_{\mathrm{r}}(\mathrm{IH})$ & $0.0183-0.0297$ \\
\hline
\end{tabular}

Table 1. The $3 \sigma$ values of the global fit oscillation parameters, the mass-squared differences and mixing angles [33].

generation mechanism. If light Majorana neutrinos are the only source of $0 \nu \beta \beta$ transition, we can express the half-life as

$$
\frac{1}{T_{1 / 2}^{0 \nu}}=G_{01}^{0 \nu}\left|\mathcal{M}_{\nu}^{0 \nu}\right|^{2}\left|\eta_{\nu}\right|^{2}=G_{01}^{0 \nu}\left|\frac{\mathcal{M}_{\nu}^{0 \nu}}{m_{e}}\right|^{2}\left|m_{e e}^{\nu}\right|^{2},
$$

which contains three important factors: i) the $\operatorname{NME~} \mathcal{M}_{\nu}^{0 \nu}$, ii) the phase space factor $G_{01}^{0 \nu}$, and iii) a dimensionless particle physics parameter - a measure of lepton number violation $\eta_{\nu} \equiv m_{e e}^{\nu} / m_{e}$ which is a function of neutrino masses, mixing angles, and CP phases,

$$
\begin{aligned}
\left|m_{e e}^{\nu}\right|^{2} & =|| U_{e 1}^{2}\left|m_{1}+\right| U_{e 2}^{2}\left|m_{2} e^{i \alpha}+\right| U_{e 3}^{2}\left|m_{3} e^{i \beta}\right| \\
& =\left|c_{s}^{2} c_{r}^{2} m_{1}+s_{s}^{2} c_{r}^{2} m_{2} e^{i \alpha}+s_{r}^{2} m_{3} e^{i \beta}\right|^{2},
\end{aligned}
$$

where $m_{i}$ are the mass eigenvalues of light neutrinos, $\alpha, \beta$ are the two Majorana CP phases, and $U_{e i}$ are the elements of PMNS mixing matrix. We present the variation of $\left|m_{e e}^{\nu}\right|$, due to the unknown Majorana CP phases, as a function of the lightest neutrino mass $\left(m_{1}\right.$ for $\mathrm{NH}$ and $m_{3}$ for $\left.\mathrm{IH}\right)$ in figure 1 . The notation of mixing angles is defined as $\left(c_{\alpha}, s_{\alpha}\right) \equiv$ $\left(\cos \theta_{\alpha}, \sin \theta_{\alpha}\right)$ where $\theta_{\mathrm{a}} \equiv \theta_{23}$ is the atmospheric mixing angle, $\theta_{\mathrm{r}} \equiv \theta_{13}$ the reactor mixing angle, and $\theta_{\mathrm{s}} \equiv \theta_{12}$ the solar mixing angle. In addition, the oscillation data are sensitive to two mass-squared differences, the solar mass-squared difference $\Delta m_{\mathrm{s}}^{2} \equiv m_{2}^{2}-m_{1}^{2}$ and the atmospheric mass-squared difference $\Delta m_{\mathrm{a}}^{2} \equiv\left|m_{3}^{2}-m_{1}^{2}\right|$. Since we know only the magnitude of the atmospheric mass-squared difference with its sign unknown, there exit two different mass patterns among light neutrinos: NH with $m_{1}<m_{2}<m_{3}$ and IH with $m_{3}<m_{1}<m_{2}$. The $3 \sigma$ global fit ranges for the oscillation parameters are summarized in table 1 .

The effective Majorana mass parameter of neutrinoless double beta decay that predicted by the standard mechanism is displayed in figure 1 with light-red (green) band for $\mathrm{NH}(\mathrm{IH})$, respectively. The horizontal bands are bounds from neutrinoless double beta decay measurements which can provide crucial information about the absolute scale of light neutrino mass and Majorana CP phases. In addition, the mass scale can also be bounded from i) the measurement of beta decay parameter $m_{\beta}=\sqrt{\sum_{i} U_{e i}^{2} m_{i}}$ for which the existing 


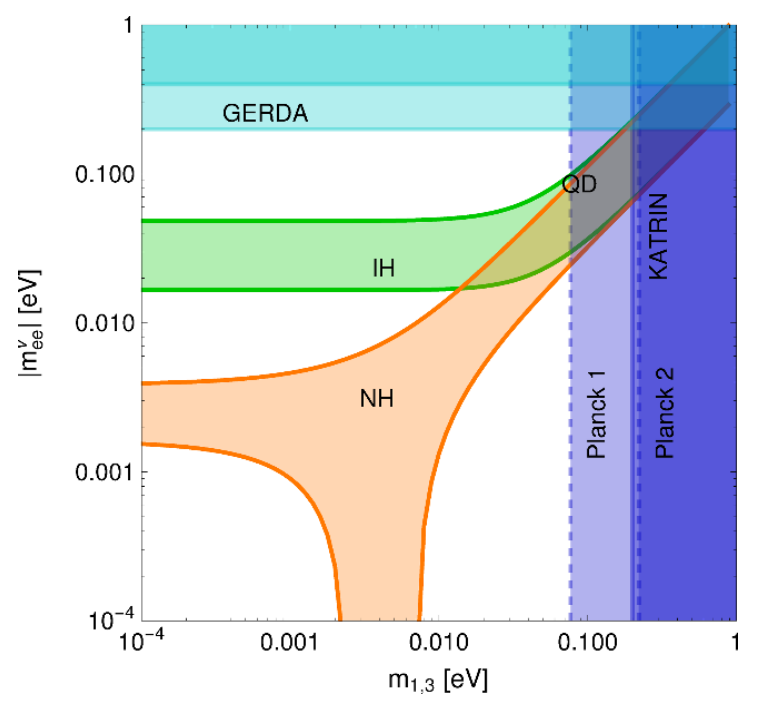

Figure 1. Effective Majorana mass for neutrinoless double beta decay within the standard mechanism as a function of the lightest neutrino mass, $m_{1}$ for $\mathrm{NH}$ (yellow band) and $m_{3}$ for IH (green band). The oscillation parameters are varied within the $3 \sigma$ ranges taken from ref. [33] while the Majorana CP phases $\alpha$ and $\beta$ are treated as free parameters. The horizontal band are excluded regions of the effective Majorana mass parameter $m_{e e}^{\nu}$ by current neutrinoless double beta decay measurement (GERDA), while the vertical bands are excluded regions of the mass scale by beta decay (KARTRIN) and cosmological observations (Planck).

bound is $m_{\beta}<0.2 \mathrm{eV}$ from KATRIN [34], ii) The limits on the effective Majorana mass parameter from various isotopes [30],

$$
\begin{array}{ll}
\left|m_{e e}^{\nu}\right| \leq(0.22 \sim 0.53) \mathrm{eV} & \text { For }{ }^{76} \mathrm{Ge}, \\
\left|m_{e e}^{\nu}\right| \leq(0.36 \sim 0.90) \mathrm{eV} & \text { For }{ }^{100} \mathrm{Mo}, \\
\left|m_{e e}^{\nu}\right| \leq(0.27 \sim 1.00) \mathrm{eV} & \text { For }{ }^{130} \mathrm{Te}, \\
\left|m_{e e}^{\nu}\right| \leq(0.15 \sim 0.35) \mathrm{eV} & \text { For }{ }^{136} \mathrm{Xe} .
\end{array}
$$

iii) Cosmological observations which provide constraints on the sum of light neutrino masses $m_{\Sigma} \equiv \sum_{i} m_{i}$. They have been shown as vertical bands in figure 1 . Note that these constraints have only touched down to the quasi-degenerate region, $m_{1} \simeq m_{2} \simeq$ $m_{3}$ and $m_{\text {lightest }}>\sqrt{\Delta m_{\mathrm{a}}^{2}}$, with the beta-decay and cosmological constraints approximately at $\sum_{i} m_{i} / 3 \simeq m_{\text {lightest }} \simeq m_{\beta} \geq\left|m_{e e}^{\nu}\right|$ which is the same for both hierarchies. The current bound on the sum of light neutrino mass is $m_{\Sigma}<0.23 \mathrm{eV}$ derived from Planck+WP+highL+BAO data (Planck1) at 95\% C.L. while $m_{\Sigma}<1.08 \mathrm{eV}$ from Planck+ WP+highL (Planck2) at 95\% C.L. [35]. It is claimed that the latest cosmological constraint can be approximated by gaussian distribution, $m_{\Sigma}=22 \pm 62 \mathrm{meV}$ [32]. At $1 \sigma$ C.L., the mass sum is smaller than $84 \mathrm{meV}$ which is below the smallest value for IH. In this sense, the NH is favored. There is no hope to probe the normal hierarchy within the standard mechanism, even for next-generation experiments. On the contrary, if there is new physics contribution in addition to the standard mechanism, the neutrinoless double beta decay can be sizable. In the following part, we discuss one possibility of LRSM with 
Type-II seesaw dominance which is fully predictable and can be tested at next-generation experiments.

\section{Effect of new physics contribution}

In this section we briefly discuss a simple left-right symmetric model as the source of new physics that can contribute to neutrinoless double beta decay and is fully predictable. The left-right symmetric model [1-6] is based on the gauge group $\mathrm{SU}(2)_{\mathrm{L}} \times \mathrm{SU}(2)_{\mathrm{R}} \times \mathrm{U}(1)_{B-L^{-}}$ omitting $\mathrm{SU}(3)_{C}$ structure for simplicity-where $\mathrm{SU}(2)_{\mathrm{L}}$ interchanges with $\mathrm{SU}(2)_{\mathrm{R}}$ under parity. The quarks and leptons individually transform as

$$
\begin{aligned}
Q_{\mathrm{L}} & =\left(\begin{array}{c}
u_{\mathrm{L}} \\
d_{\mathrm{L}}
\end{array}\right) \equiv\left[2,1, \frac{1}{3}\right], & Q_{\mathrm{R}}=\left(\begin{array}{l}
u_{\mathrm{R}} \\
d_{\mathrm{R}}
\end{array}\right) \equiv\left[1,2, \frac{1}{3}\right], \\
\ell_{\mathrm{L}} & =\left(\begin{array}{c}
\nu_{\mathrm{L}} \\
e_{\mathrm{L}}
\end{array}\right) \equiv[2,1,-1], & \ell_{\mathrm{R}}=\left(\begin{array}{c}
\nu_{\mathrm{R}} \\
e_{\mathrm{R}}
\end{array}\right) \equiv[1,2,-1] .
\end{aligned}
$$

Spontaneous symmetry breaking is implemented as usual with the help of triplet and bidoublet Higgs fields,

$$
\Delta_{\mathrm{L}, \mathrm{R}} \equiv\left(\begin{array}{cc}
\delta_{\mathrm{L}, \mathrm{R}}^{+} / \sqrt{2} & \delta_{\mathrm{L}, \mathrm{R}}^{++} \\
\delta_{\mathrm{L}, \mathrm{R}}^{0} & -\delta_{\mathrm{L}, \mathrm{R}}^{+} / \sqrt{2}
\end{array}\right), \quad \Phi \equiv\left(\begin{array}{cc}
\phi_{1}^{0} & \phi_{2}^{+} \\
\phi_{1}^{-} & \phi_{2}^{0}
\end{array}\right),
$$

with quantum numbers $(3,1,2),(1,3,2)$, and $(2,2,0)$, respectively. The bidoublet connects left- and right-handed fermions to provide Dirac mass term while the triplet provides Majorana mass term to left- or right-handed neutrinos. After symmetry breaking, the neutrino mass matrix can be written as,

$$
M_{\nu}=\left(\begin{array}{cc}
M_{\mathrm{L}} & m_{\mathrm{D}} \\
m_{\mathrm{D}}^{T} & M_{\mathrm{R}}
\end{array}\right)
$$

where, $M_{\mathrm{L}}=f_{\mathrm{L}}\left\langle\Delta_{\mathrm{L}}\right\rangle=f v_{\mathrm{L}}\left(M_{\mathrm{R}}=f_{\mathrm{R}}\left\langle\Delta_{\mathrm{R}}\right\rangle=f v_{\mathrm{R}}\right)$ is the LH (RH) Majorana masses for light (heavy) neutrinos while $m_{\mathrm{D}}=y v_{1}+\tilde{y} v_{2}$ is the Dirac neutrino mass term connecting light and heavy neutrinos. The parity is first broken by $v_{\mathrm{R}}$, the vacuum expectation value $(\mathrm{VEV})$ of the right-handed triplet $\Delta_{\mathrm{R}}$, reducing $\mathrm{SU}(2)_{\mathrm{L}} \times \mathrm{SU}(2)_{\mathrm{R}} \times \mathrm{U}(1)_{Y}$ to the $\mathrm{SM}$ gauge structure $\mathrm{SU}(2)_{\mathrm{L}} \times \mathrm{U}(1)_{Y}$ which is further broken by the VEV of the bidoublet $\Phi$. This symmetry breaking sequence naturally requires $v_{\mathrm{R}}$ to be much larger than the electroweak scale. On the other hand, the VEV $v_{\mathrm{R}}$ of the left-handed triple $\Delta_{\mathrm{L}}$ is small. With natural Yukawa couplings, the mass matrix elements of $M_{\nu}$ have a hierarchical structure, $M_{\mathrm{R}} \gg m_{\mathrm{D}} \gg M_{\mathrm{L}}$. The light neutrino mass matrix can be obtained via Type-I+II seesaw mechanism,

$$
m_{\nu}=M_{\mathrm{L}}-m_{\mathrm{D}} M_{\mathrm{R}}^{-1} m_{\mathrm{D}}^{T}=m_{\nu}^{I I}+m_{\nu}^{I} .
$$

The Majorana nature of the light and heavy neutrinos, arising from the presence of the scalar triplets $\Delta_{\mathrm{L}, \mathrm{R}}$ with charge $B-L=2$, implies that they can mediate neutrinoless double beta decay [7, 24-29], as shown in figure 2. The left- and right-handed currents 

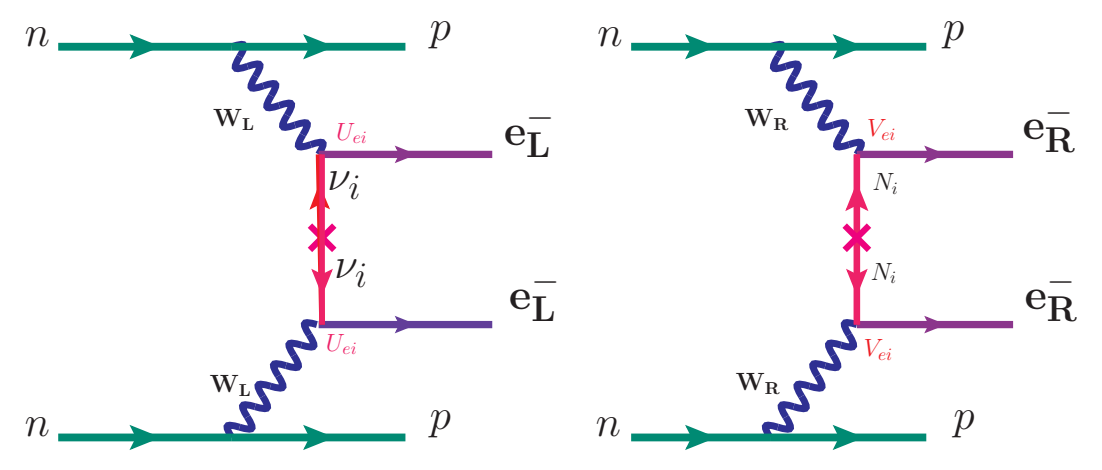

Figure 2. Feynman diagrams of neutrinoless double beta decay processes. The left-panel is for purely left-handed current contribution by the standard mechanism while the right-panel is for purely right-handed current contribution by LRSM with Type-II seesaw dominance.

contribute independently without interference. The other diagrams contributing to neutrinoless double beta decay via doubly charged scalar triplet exchange, $\lambda$-diagram involving $W_{\mathrm{L}}-W_{\mathrm{R}}$ mediation and $\eta$-diagram are suppressed, for details, see refs. [25, 28, 29].

\subsection{Type-II seesaw dominance}

As shown in (3.3), the light neutrino mass matrix receives two independent contributions from Type-I and Type-II seesaw mechanisms. Although the left- and right-handed Yukawa/mass matrices are connected with each other, $M_{\mathrm{L}} \propto M_{\mathrm{R}}$, due to parity, the Dirac mass matrix $m_{\mathrm{D}}$ is independent of the others. Experimentally, we have measured the oscillation parameters of the light neutrinos. The information is not enough to constrain both $M_{\mathrm{L}}\left(M_{\mathrm{R}}\right)$ and $m_{\mathrm{D}}$. While the light neutrino mass matrix $m_{\nu}$ can be reconstructed, the heavy one $M_{\mathrm{R}}$ is out of our knowledge. On the other hand, both the light and heavy neutrino mass matrices are involved in the neutrinoless double beta decay process as shown in figure 2. It is difficult to make a specific prediction unless we can somehow know the heavy neutrino mass matrix $M_{\mathrm{R}}$. Fortunately, Type-II seesaw dominance can save the situation here [7, 25, 27, 28, 36].

With Type-II seesaw dominance, which can be realized by suppressing the Dirac mass term $m_{\mathrm{D}}$, the mass matrices of the left- and right-handed neutrinos are proportional to each other,

$$
m_{\nu}=M_{\mathrm{L}} \propto M_{\mathrm{R}} .
$$

A direct consequence is that the left- and right-handed neutrinos share the same mixing matrix,

$$
V_{\mathrm{R}}^{\mathrm{PMNS}}=V_{\mathrm{L}}^{\mathrm{PMNS}},
$$

in the diagonal basis of charged leptons. The unmeasured right-handed neutrino mixing can then be fully reconstructed.

Actually, the equality between the left- and right-handed PMNS mixing matrices can also come from the lepton sector. To see this let us first write down the Yukawa couplings 
that are responsible for the lepton mass matrix,

$$
\bar{L}_{\mathrm{L}}\left(Y_{\ell} \Phi+Y_{\ell}^{\prime} \Phi^{\dagger}\right) L_{\mathrm{R}}+\text { h.c. }
$$

where $\Phi^{\dagger} \equiv \sigma_{2} \Phi^{*} \sigma_{2}$ is the CP mirror of the bidoublet $\Phi$ with $\tau_{2}$ being the second Pauli matrix. When the Higgs bidoublet develops a non-zero VEV,

$$
\Phi=\left(\begin{array}{ll}
v_{1} e^{i \theta_{1}} & \\
& v_{2} e^{i \theta_{2}}
\end{array}\right),
$$

the leptons receive a mass matrix,

$$
M_{\ell}=Y_{\ell} v_{2} e^{i \theta_{2}}+Y_{\ell}^{\prime} v_{1} e^{-i \theta_{1}}
$$

If the Higgs doublet fails to develop trivial CP phases or if the two Yukawa matrices $Y_{\ell}$ and $Y_{\ell}^{\prime}$ commute with each other, the lepton mass matrix can commute with its Hermitian conjugate,

$$
M_{\ell} M_{\ell}^{\dagger}=M_{\ell}^{\dagger} M_{\ell}
$$

A direct consequence is that the left- and right-handed leptons share the same mixing matrix,

$$
V_{\ell, L}=V_{\ell, R}
$$

If the neutrino mixing is trivial, $V_{\nu}=I$, then the PMNS mixing matrices come solely from the charged lepton mixing, reproducing (3.5).

For all cases, the mass eigenvalues for light as well as heavy neutrinos are related as,

$$
M_{i} \propto m_{i}
$$

where $M_{i}$ and $m_{i}$ are physical masses for light active and heavy Majorana neutrinos. Fixing $M_{\max }\left(M_{3}\right.$ for $\mathrm{NH}$ and $M_{2}$ for $\left.\mathrm{IH}\right)$, the mass relation can be expressed as,

$$
\begin{aligned}
& M_{i}=\frac{m_{i}}{m_{3}} M_{3}, \quad \text { for } \quad \mathrm{NH}, \\
& M_{i}=\frac{m_{i}}{m_{2}} M_{2}, \quad \text { for } \quad \mathrm{IH} .
\end{aligned}
$$

\section{Comparison between new physics and standard mechanism}

The inverse of half-life for a particular isotope, with new physics effects realized via a leftright symmetric model assuming Type-II seesaw dominance, contributes to neutrinoless double beta decay therefore in the following way,

$$
\begin{aligned}
\frac{1}{T_{1 / 2}^{0 \nu}} & =G_{01}^{0 \nu}\left[\left|\mathcal{M}_{\nu}^{0 \nu} \cdot \eta_{\nu}\right|^{2}+\left|\mathcal{M}_{N}^{0 \nu} \cdot \eta_{N}\right|^{2}\right] \\
& =G_{01}^{0 \nu}\left|\frac{\mathcal{M}_{\nu}^{0 \nu}}{m_{e}}\right|^{2}\left|m_{\mathrm{ee}}^{(\nu+N)}\right|^{2},
\end{aligned}
$$




\begin{tabular}{|lccc|}
\hline Isotope & $G_{01}^{0 \nu}\left(\right.$ yr. $\left.^{-1}\right)$ & $\mathcal{M}_{\nu}^{0 \nu}$ & $\mathcal{M}_{N}^{0 \nu}$ \\
\hline${ }^{76} \mathrm{Ge}$ & $5.77 \times 10^{-15}$ & $2.58-6.64$ & $233-412$ \\
${ }^{136} \mathrm{Xe}$ & $3.56 \times 10^{-14}$ & $1.57-3.85$ & $164-172$ \\
\hline
\end{tabular}

Table 2. The Phase space factor $G_{01}^{0 \nu}\left(\mathrm{yr}^{-1}\right)$ and nuclear matrix elements (NMEs) for two different isotopes ${ }^{76} \mathrm{Ge}$ and ${ }^{136} \mathrm{Xe}[37]$.

where $\left|m_{\mathrm{ee}}^{(\nu+N)}\right|^{2} \equiv\left|m_{\mathrm{ee}}^{\nu}\right|^{2}+\left|m_{\mathrm{ee}}^{N}\right|^{2}$ with explicit analytic expressions under Type-II seesaw dominance,

$$
\begin{gathered}
\left|m_{\mathrm{ee}}^{\nu}\right| \equiv\left|c_{s}^{2} c_{r}^{2} m_{1}+s_{s}^{2} c_{r}^{2} m_{2} e^{i \alpha}+s_{r}^{2} m_{3} e^{i \beta}\right| \\
\left|m_{\mathrm{ee}}^{N}\right|_{N H} \equiv \frac{C_{N}}{M_{3}}\left|c_{s}^{2} c_{r}^{2} \frac{m_{3}}{m_{1}}+s_{s}^{2} c_{r}^{2} \frac{m_{3}}{m_{2}} e^{i \alpha}+s_{r}^{2} e^{i \beta}\right|, \\
\left|m_{\mathrm{ee}}^{N}\right|_{I H} \equiv \frac{C_{N}}{M_{2}}\left|c_{s}^{2} c_{r}^{2} \frac{m_{2}}{m_{1}}+s_{s}^{2} c_{r}^{2} e^{i \alpha}+\frac{m_{2}}{m_{3}} s_{r}^{2} e^{i \beta}\right| .
\end{gathered}
$$

The coefficient $C_{N} \equiv\left\langle p^{2}\right\rangle\left(M_{W_{\mathrm{L}}} / M_{W_{\mathrm{R}}}\right)^{4}\left(g_{\mathrm{R}} / g_{\mathrm{L}}\right)^{4}$ contains the typical momentum transfer $\langle p\rangle \approx 100 \mathrm{MeV}$. Here, the lepton number violating particle physics parameters are $\eta_{\nu}$ and $\eta_{N}\left(m_{e e}^{\nu}, m_{e e}^{N}\right)$, while $G_{01}^{0 \nu}$ is the phase space factor, and $\mathcal{M}_{\nu}^{0 \nu}\left(\mathcal{M}_{N}^{0 \nu}\right)$ the NMEs derived for virtual light and heavy particle exchanged diagram, respectively, whose values can be found in table 2. The $\mathrm{SU}(2)_{\mathrm{L}}$ and $\mathrm{SU}(2)_{\mathrm{R}}$ coupling constants $g_{\mathrm{L}}$ and $g_{\mathrm{R}}$, respectively, need not to be the same for LRSM. In addition to an overall factor $C_{N} / M_{3}$ for $\mathrm{NH}$ or $C_{N} / M_{2}$ for $\mathrm{IH}, m_{e e}^{N}$ share the same set of input parameters as $m_{e e}^{\nu}$. To estimate the lepton number violating parameter arising from right-handed current, It is necessary to know oscillation parameters, the Majorana CP phases, and the mass scales.

- Oscillation parameters: neutrino oscillation experiments has measured the two masssquared differences and the three mixing angles. Nevertheless only two mixing angles, the reactor mixing angle $\theta_{\mathrm{r}}$ and the solar mixing angle $\theta_{\mathrm{s}}$, are relevant in the effective Majorana mass parameter. The error of these four oscillation parameters can introduce uncertainty in the prediction of $\left|m_{e e}^{\nu}\right|$, especially the solar mixing angle $\theta_{\mathrm{s}}[38]$. With the current global fit [33], the upper limit on the minimal half-life for IH varies by a factor of 2.5 at $3 \sigma$ C.L. Fortunately, the same set of oscillation parameters is shared by reactor neutrino experiments and hence can be precisely measured by next-generation medium-baseline experiments like JUNO [39]. The uncertainty from oscillation parameters can be reduced to below $1 \%$ which is essentially negligible [30, 31].

- $C P$ Phases: Although the Dirac CP phase $\delta_{\mathrm{D}}$ also enters the effective Majorana mass parameter $\left|m_{e e}^{\nu}\right|$ and $\left|m_{e e}^{N}\right|$, it will not manifest itself in the predicted values. This is because it is always associated with one of the two Majorana CP phases $\alpha$ and $\beta$ which are completely unknown. For simplicity, the Dirac CP phase has been omitted in the expressions. The variation of $\alpha$ and $\beta$ renders the predicted values of $\left|m_{e e}^{\nu}\right|$ 


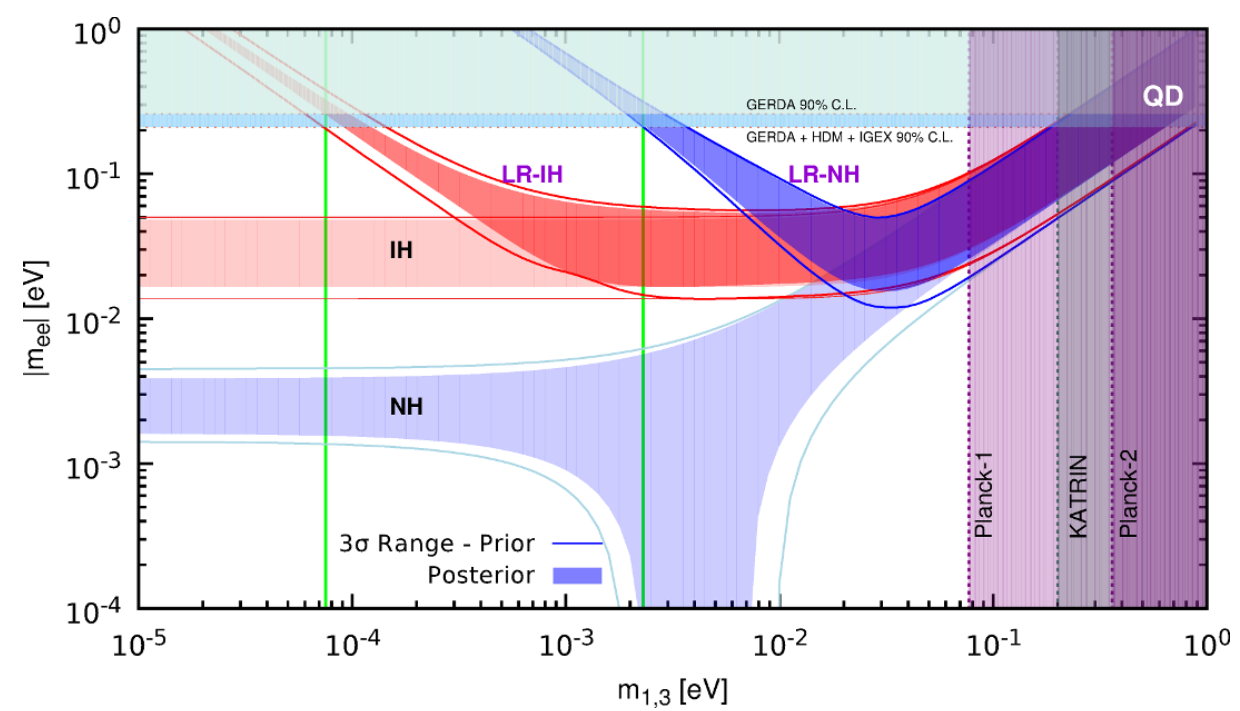

Figure 3. The SM and LRSM-Type II contributions to the effective Majorana mass parameter $\left|m_{e e}\right|$ as a function of the lightest neutrino mass, $m_{1}$ for $\mathrm{NH}$ (dark blue band) and $m_{3}$ for IH (dark red band), within the $3 \sigma$ ranges of both prior from current global fit (band) and posterior after including JUNO (enveloping curve). The horizontal bands are the current experimental bounds on the effective mass of neutrinoless double beta decay, from which lower limits (vertical green lines on the left) on the lightest neutrino mass can be extracted. The vertical bands on the right are beta decay and cosmological constrains excluding most part of quasi-degenerate region.

and $\left|m_{e e}^{N}\right|$ to span in a wide band. In appendix A, we generalize the geometrical picture of obtaining the minimal and maximal values within standard mechanism to LRSM-Type II seesaw dominance.

- Mass Scales: in addition to oscillation parameters and CP phases, the mass scales of light and heavy neutrinos are still unknown yet. For light neutrinos, we fix the mass scale by hand since there is no measurement at all. On the other hand, the contribution from heavy neutrinos involve two mass scales, the heavy neutrino and $W_{\mathrm{R}}$ masses. First, we need to fix the heavy neutrino mass scales by fixing $M_{3}$ for $\mathrm{NH}$ or $M_{2}$ for IH. Second, the $W_{\mathrm{R}}$ mass also enters through $C_{N}$. When combined, the net effect is an overall factor, $1 /\left(M_{W_{\mathrm{R}}}^{4} M_{3}\right)$ for $\mathrm{NH}$ or $1 /\left(M_{W_{\mathrm{R}}}^{4} M_{2}\right)$ for IH. A natural realization is $M_{2,3}$ should be roughly of the same scale as $M_{W_{\mathrm{R}}}$. In other words, the low-energy neutrino phenomenology is highly related with collider signature. If the di-boson excess [8] observed at ATLAS really comes from LRSM with mass $M_{W_{\mathrm{R}}} \approx$ $2 \mathrm{TeV}$, its contribution to low energy neutrino phenomenology is large enough to be observed by next-generation neutrinoless double beta decay experiments.

We show in figure 3 the lepton number violating effective Majorana mass parameters vs lightest neutrino mass for ${ }^{76} \mathrm{Ge}$ which is used by GERDA [13]. For comparison, we show both the total contribution and the one from standard mechanism without new physics. The numerical analysis has been performed using $3 \sigma$ prior and posterior distributions from current global fit and the one improved with JUNO [39]. As studied in [30] the combination 
of short- and medium-baseline reactor experiments can achieve precise measurement of the oscillation parameters involved in neutrinoless double beta decay. Here, we adopt the same configuration of JUNO with $53 \mathrm{~km}$ of baseline, $20 \mathrm{kt}$ of detector, $36 \mathrm{GW}$ of thermal power, 6 years of running (300 effective days for each year), and energy resolution $3 \% / \sqrt{E / \mathrm{MeV}}$. This roughly reproduce the results of the JUNO yellow book [39]. It is clearly shown in figure 3 that the uncertainty from oscillation parameters has significant effect on the prediction of the effective Majorana mass parameter within not only the standard mechanism but also the total contribution.

In figure 3 we have fixed $M_{W_{\mathrm{R}}} \simeq 2 \mathrm{TeV}, g_{\mathrm{R}} \approx 2 / 3 g_{\mathrm{L}}$, and $M_{N}=1 \mathrm{TeV}$ which can satisfy both ATLAS [8] and CMS [9] excesses. With this typical mass assignment indicated by collider signature, the contribution from new physics is not small and actually can dominate over the standard mechanism in low mass region. In the quasi-degenerate region, there is no big difference between the total contribution and the standard mechaism. As the mass scale of light neutrinos decreases, the new physics contribution keeps growing and stops the total one from decreasing to zero for both hierarchies. The minimal value is within the sensitivity region of next-generation neutrinoless double beta decay experiments which can touch down to around $0.01 \mathrm{eV}$. When the smallest mass eigenvalue $m_{1,3}$ of the light neutrinos further decreases, the new physics contribution dominates and can finally saturate the current experimental bound placed by GERDA [13], Heidelberg-Moscow [40], and IGEX [41]. Since no experiment has observed neutrinoless double beta decay, we can derive lower limit on the absolute scale of light neutrinos,

$$
\begin{array}{ll}
m_{1}>2.3 \mathrm{meV} & \text { for } \mathrm{NH}, \\
m_{3}>0.075 \mathrm{meV} & \text { for } \mathrm{IH} .
\end{array}
$$

as indicated by solid vertical lines in figure 3. This is totally different from the standard mechanism based on which neutrinoless double beta decay experiments can only provide upper limits. For IH, the standard mechanism cannot provide lower limit which may be possible for NH. On the contrary, the LRSM-Type II contribution can place both lower and upper limits on the mass scale of light neutrinos.

The lower limits obtained from current neutrinoless double beta decay measurements are consistent with the cosmological constraint on the sum of light neutrino masses [42-44],

$$
\begin{array}{ll}
m_{\Sigma}<84 \mathrm{meV} & (1 \sigma \text { C.L. }), \\
m_{\Sigma}<146 \mathrm{meV} & (2 \sigma \text { C.L. }), \\
m_{\Sigma}<208 \mathrm{meV} & (3 \sigma \text { C.L. }) .
\end{array}
$$

For clarity, we replot the effective Majorana mass parameter in figure 4 as a fuction of the mass sum. It clearly shows that at more than $1 \sigma$ C.L., NH is favored over IH. If so, the low-energy neutrinoless double beta decay cannot be observed even at next-generation experiments, if no new physics is present. Fortunately, the diboson excess observed at ATLAS provides a timely way out. 


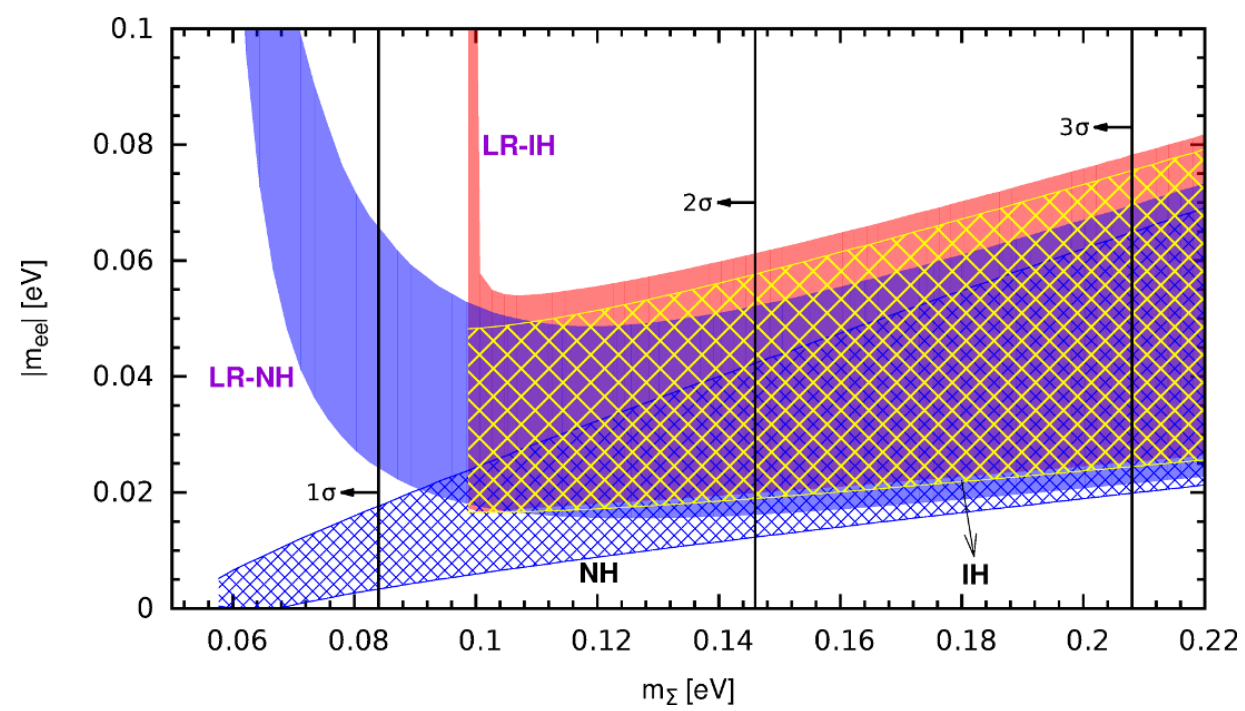

Figure 4. Allowed region of $\left|m_{e e}\right|$ as a function of the light neutrino mass sum $m_{\Sigma}$. The standard mechanism contribution is plotted with hatched bands, the dense blue one for $\mathrm{NH}$ and sparse yellow one for IH, while the new physics contribution with shaded bands, the blue one for LR-NH and the red one for LR-IH. The comparison between SM and LRSM-Type II seesaw contributions to the effective Majorana mass parameter $\left|m_{e e}\right|$ as a function of the mass sum has been presented using JUNO data and bound from cosmology. The limits on mass sum at $1 \sigma, 2 \sigma$ and $3 \sigma$ C.L., $m_{\Sigma}<84 \mathrm{meV}, m_{\Sigma}<146 \mathrm{meV}$, and $m_{\Sigma}<208 \mathrm{meV}$, respectively, are shown as vertical lines.

\section{Separating the SM and LRSM contributions}

In LRSM with Type-II seesaw, the two contributions are linearly combined as shown in (4.1). Using two isotopes, $\left(A_{i}, Z_{i}\right)$ and $\left(A_{j}, Z_{j}\right)$, it is possible to separate the SM and non-interfering new physics contributions [37, 45],

$$
\begin{aligned}
\left|m_{e e}^{\nu}\right|^{2} & =\frac{\left|M_{N, j}^{0 \nu}\right|^{2} / T_{i} G_{i}-\left|M_{N, i}^{0 \nu}\right|^{2} / T_{j} G_{j}}{\left|M_{\nu, i}^{0 \nu}\right|^{2}\left|M_{N, j}^{0 \nu}\right|^{2}-\left|M_{N, i}^{0 \nu}\right|^{2}\left|M_{\nu, j}^{0 \nu}\right|^{2}}, \\
\left|m_{e e}^{N}\right|^{2} & =\frac{\left|M_{\nu, i}^{0 \nu}\right|^{2} / T_{j} G_{j}-\left|M_{\nu, j}^{0 \nu}\right|^{2} / T_{i} G_{i}}{\left|M_{\nu, i}^{0 \nu}\right|^{2}\left|M_{N, j}^{0 \nu}\right|^{2}-\left|M_{N, i}^{0 \nu}\right|^{2}\left|M_{\nu, j}^{0 \nu}\right|^{2}} .
\end{aligned}
$$

However, there is sizable uncertainty in the theoretical calculation of the nuclear matrix elements which implies that the extracted effective Majorana mass parameters $\left|m_{e e}^{\nu}\right|$ and $\left|m_{e e}^{N}\right|$ have uncertainties.

Fortunately, the theoretical uncertainties can be significantly reduced by taking the ratios between nuclear matrix elements as well as between half-lifes of different isotopes. These ratios can be used to obtain the ratio of new physics and standard mechanism effective Majorana mass parameters,

$$
\left|\frac{m_{e e}^{N}}{m_{e e}^{\nu}}\right|^{2}=\left|\frac{M_{\nu, j}^{0 \nu}}{M_{N, i}^{0 \nu}}\right|^{2} \frac{T_{i} G_{i}}{T_{j} G_{j}} \frac{\left|\frac{M_{\nu, i}^{0 \nu}}{M_{\nu, j}^{0 \nu}}\right|^{2}-\frac{T_{j} G_{j}}{T_{i} G_{i}}}{\left|\frac{M_{N, j}^{0 \nu}}{M_{N, i}^{0 \nu}}\right|^{2}-\frac{T_{i} G_{i}}{T_{j} G_{j}}} .
$$



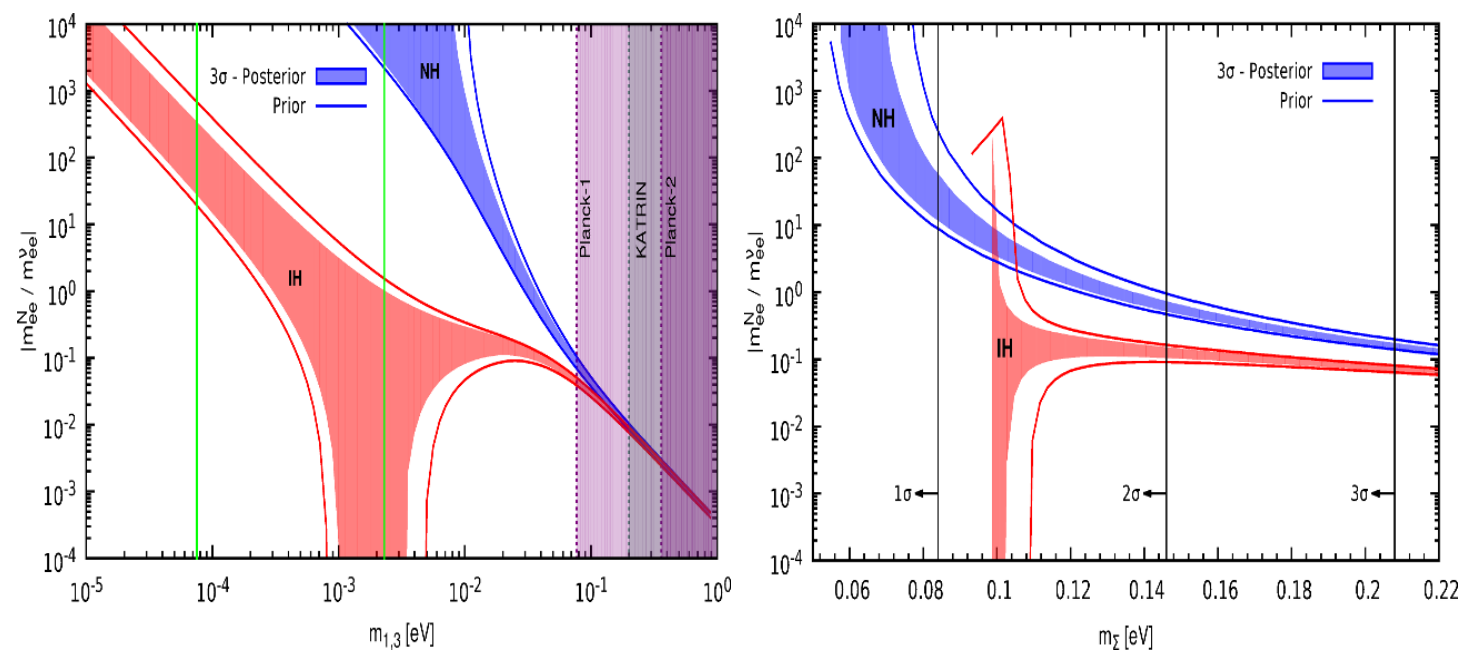

Figure 5. Ratio between effective Majorana mass parameters $\left|m_{e e}^{N} / m_{e e}^{\nu}\right|$ as a function of lightest neutrino mass, $m_{1}$ for $\mathrm{NH}$ and $m_{3}$ for $\mathrm{IH}$ in the left. The vertical green lines comes from the saturation limit in figure 3. In the right, we show the ratio $\left|m_{e e}^{N} / m_{e e}^{\nu}\right|$ as a function of the mass sum $m_{\Sigma}$ together with recent cosmological constraints. Here $\left|m_{e e}^{\nu}\right|$ is the standard mechanism contribution with $W_{\mathrm{L}}-W_{\mathrm{L}}$ mediation while $\left|m_{e e}^{N}\right|$ the LRSM-Type-II seesaw contribution via $W_{\mathrm{R}^{-}}$ $W_{\mathrm{R}}$ mediation.

One can therefore expect that this ratio will be more precisely determined than the individual effective Majorana mass parameters.

In figure 5, we show the ratio $\left|m_{e e}^{N} / m_{e e}^{\nu}\right|$ as a function of the lightest neutrino mass in the left panel. Its value can span several orders of magnitude. In the quasi-degenerate region, the ratio is typically below 0.1 . For $\mathrm{NH}$, it grows with decreasing mass scale. On the other hand it can experience a short range, $1 \mathrm{meV} \lesssim m_{3} \lesssim 4 \mathrm{meV}$, of touching down to the bottom, although the maximal value keeps growing with decreasing $m_{3}$. Across the whole range, the ratio for $\mathrm{NH}$ is always larger than its value for IH. Together with the lower limit on the lightest mass extracted from current neutrinoless double beta decay measurements, as depicted in figure 3 , the ratio is constrained to be smaller than $10^{3}$ for $\mathrm{IH}$ while it can go well above $10^{4}$ for NH. If neutrinoless double beta decay are observed with two isotopes at future experiments to reconstruct the ratio $\left|m_{e e}^{N} / m_{e e}^{\nu}\right|$, it is possible to determine the neutrino mass hierarchy.

According to the definition (4.2) of effective Majorana mass parameters, the contribution of the right-handed sector actually can be separated from light neutrino parameters since it only appears as an overall factor $C_{N} / M_{3}$ for $\mathrm{NH}$ or $C_{N} / M_{2}$ for IH. From the measured ratio (5.2) we can reconstruct,

$$
\begin{gathered}
\frac{\left|c_{s}^{2} c_{r}^{2} m_{1}+s_{s}^{2} c_{r}^{2} m_{2} e^{i \alpha}+s_{r}^{2} m_{3} e^{i \beta}\right|}{\left|c_{s}^{2} c_{r}^{2} \frac{m_{3}}{m_{1}}+s_{s}^{2} c_{r}^{2} \frac{m_{3}}{m_{2}} e^{i \alpha}+s_{r}^{2} e^{i \beta}\right|}=\left|\frac{m_{e e}^{N}}{m_{e e}^{\nu}}\right| \frac{M_{3}}{C_{N}}, \\
\frac{\left|c_{s}^{2} c_{r}^{2} m_{1}+s_{s}^{2} c_{r}^{2} m_{2} e^{i \alpha}+s_{r}^{2} m_{3} e^{i \beta}\right|}{\left|c_{s}^{2} c_{r}^{2} \frac{m_{2}}{m_{1}}+s_{s}^{2} c_{r}^{2} e^{i \alpha}+\frac{m_{2}}{m_{3}} s_{r}^{2} e^{i \beta}\right|}=\left|\frac{m_{e e}^{N}}{m_{e e}^{\nu}}\right| \frac{M_{2}}{C_{N}},
\end{gathered}
$$


which can be compared with collider measurements on the right-handed sector parameters contained in $M_{2,3} / C_{N}$. This can be used to add another input to determine the Majorana CP phases. The degeneracy between $\alpha$ and $\beta$ can then be eliminated.

\section{Conclusions}

We have shown that new physics contributions to neutrinoless double beta decay induced by right-handed current can saturate the present experimental bound. Then, the neutrinoless double beta decay experiment is still possible to see a signal for $\mathrm{NH}$ which together with small mass scale is favored by the recent bound on $m_{\Sigma}$ from cosmology. In comparison, if only the standard mechanism contributes, it is difficult to see a signal of neutrinoless double beta decay even at next-generation experiments. Fortunately, collider signature from ATLAS and CMS has indicated heavy gauge boson $W^{\prime}$ around $2 \mathrm{TeV}$, pointing to a timely way out.

We studied a simple framework of LRSM to provide the new physics effects to neutrinoless double beta decay. The neutrino mass mechanism is governed by Type-II seesaw dominance such that the mass eigenvalues and mixing matrices of light and heavy neutrinos are correlated with each other. To illustrate the idea, we carried out numerical estimation for $M_{W_{\mathrm{R}}} \approx 2 \mathrm{TeV}$ in order to show the typical distribution.

We derived the corresponding lower limits on the absolute scale of light neutrinos, $m_{1} \gtrsim 2.3 \mathrm{meV}$ for $\mathrm{NH}$ and $m_{3} \gtrsim 0.075 \mathrm{meV}$ for $\mathrm{IH}$ by saturating the limits set by current neutrinoless double beta decay experiments. Different from the standard mechanism, where only upper limit on the mass scale can be extracted from neutrinoless double beta decay measurements, LRSM-Type II seesaw allows both upper and lower limits. In addition, the total effective Majorana mass parameter is bounded from below and well within the sensitivity reach of the next-generation experiments. To distinguish the two non-interfering contributions from the light and heavy neutrinos, we take ratios between the half-lifes and nuclear matrix elements for two isotopes. In this way, the theoretical uncertainties in the calculation of nuclear matrix elements can be avoided. Once measured and LRSMType II seesaw is confirmed, the ratio $\left|m_{e e}^{N} / m_{e e}^{\nu}\right|$ can be interpreted to distinguish the light neutrino mass hierarchy. Further, supplemented with collider measurements of the right-handed sector parameters, the two Majorana CP phases $\alpha$ and $\beta$ can be uniquely determined without degeneracy.

\section{Acknowledgments}

The work of SP is partially supported by the Department of Science and Technology, Govt. of India under the financial grant SB/S2/HEP-011/2013 and by the Max Planck Society in the project MANITOP.

\section{A Maximum and minimum values for Effective Majorana mass pa- rameter}

Given mass eigenvalues and mixing angles, the effective Majorana mass parameter can still vary due to the two unknown Majorana CP phases. A geometric picture [46] has been 
developed to obtain the maximal and minimal values analytically. This can still apply when Type-II seesaw contribution from LRSM is also included.

As a result of (3.5) within Type-II seesaw dominance, there are two contributions to the effective Majorana mass parameter,

$$
\begin{aligned}
& \left|m_{e e}^{\nu}\right|^{2}=\left|c_{s}^{2} c_{r}^{2} m_{1}+s_{s}^{2} c_{r}^{2} m_{2} e^{i \alpha}+s_{r}^{2} m_{3} e^{i \beta}\right|^{2}, \\
& \left|m_{e e}^{N}\right|^{2}=\left|c_{s}^{2} c_{r}^{2} \widetilde{m}_{1}+s_{s}^{2} c_{r}^{2} \widetilde{m}_{2} e^{i \alpha}+s_{r}^{2} \widetilde{m}_{3} e^{i \beta}\right|^{2},
\end{aligned}
$$

with concrete form of $\widetilde{m}_{i}$ to be found in the text. The interesting feature is that, in Type-II seesaw, the same set of mixing parameters, including the two Majorana CP phases $\alpha$ and $\beta$, the two mixing angles $\theta_{\mathrm{r}}$ and $\theta_{\mathrm{a}}$, as well as the Dirac CP phase $\delta_{\mathrm{D}}$, are shared between the two contributions. It provides a possibility of using the same geometrical picture to get the minimal and maximal values of $\left|m_{e e}\right|$ due to the variation of $\alpha$ and $\beta$. For convenience, let us redefine $\left|m_{e e}^{\nu}\right|$ and $\left|m_{e e}^{N}\right|$ as,

$$
\begin{aligned}
& \left|m_{e e}^{\nu}\right|^{2} \equiv\left|f_{1}+f_{2} e^{i \alpha}+f_{3} e^{i \beta}\right|^{2}, \\
& \left|m_{e e}^{N}\right|^{2} \equiv\left|F_{1}+F_{2} e^{i \alpha}+F_{3} e^{i \beta}\right|^{2} .
\end{aligned}
$$

When expanded, they can be written in terms of real functions,

$$
\begin{aligned}
& \left|m_{e e}^{\nu}\right|^{2}=\sum_{i} f_{i}^{2}+2 f_{1} f_{2} \cos \alpha+2 f_{1} f_{3} \cos \beta+2 f_{2} f_{3} \cos (\alpha-\beta), \\
& \left|m_{e e}^{N}\right|^{2}=\sum_{i} F_{i}^{2}+2 F_{1} F_{2} \cos \alpha+2 F_{1} F_{3} \cos \beta+2 F_{2} F_{3} \cos (\alpha-\beta) .
\end{aligned}
$$

Both contributions share the same functional form of $\alpha$ and $\beta$. After combination, the functional form remains,

$$
\left|m_{e e}\right|^{2}=\sum_{i}\left(f_{i}^{2}+F_{i}^{2}\right)+2 G_{1} G_{2} \cos \alpha+2 G_{1} G_{3} \cos \beta+2 G_{2} G_{3} \cos (\alpha-\beta),
$$

with,

$$
\begin{aligned}
G_{1} G_{2} & \equiv f_{1} f_{2}+F_{1} F_{2}, \\
G_{1} G_{3} & \equiv f_{1} f_{3}+F_{1} F_{3}, \\
G_{2} G_{3} & \equiv f_{2} f_{3}+F_{2} F_{3} .
\end{aligned}
$$

The redefined elements $G_{i}$ can be readily solved,

$$
\begin{aligned}
& G_{1}=\sqrt{\frac{\left(f_{1} f_{2}+F_{1} F_{2}\right)\left(f_{1} f_{3}+F_{1} F_{3}\right)}{f_{2} f_{3}+F_{2} F_{3}}}, \\
& G_{2}=\sqrt{\frac{\left(f_{1} f_{2}+F_{1} F_{2}\right)\left(f_{2} f_{3}+F_{2} F_{3}\right)}{f_{1} f_{3}+F_{1} F_{3}}}, \\
& G_{3}=\sqrt{\frac{\left(f_{1} f_{3}+F_{1} F_{3}\right)\left(f_{2} f_{3}+F_{2} F_{3}\right)}{f_{1} f_{2}+F_{1} F_{2}}} .
\end{aligned}
$$


The combined effective electron mass can be formulated as,

$$
\left|m_{e e}\right|^{2}=\sum_{i}\left(f_{i}^{2}+F_{i}^{2}-G_{i}^{2}\right)+\left|G_{1}+G_{2} e^{i \alpha}+G_{3} e^{i \beta}\right|^{2} .
$$

Then, the geometrical picture of finding the minimal and maximal values by varying $\alpha$ and $\beta$ as elaborated in [46] can readily apply to the last term in (A.7).

Open Access. This article is distributed under the terms of the Creative Commons Attribution License (CC-BY 4.0), which permits any use, distribution and reproduction in any medium, provided the original author(s) and source are credited.

\section{References}

[1] R.N. Mohapatra and J.C. Pati, A Natural Left-Right Symmetry, Phys. Rev. D 11 (1975) 2558 [INSPIRE].

[2] J.C. Pati and A. Salam, Lepton Number as the Fourth Color, Phys. Rev. D 10 (1974) 275 [Erratum ibid. D 11 (1975) 703] [INSPIRE].

[3] G. Senjanović and R.N. Mohapatra, Exact Left-Right Symmetry and Spontaneous Violation of Parity, Phys. Rev. D 12 (1975) 1502 [inSPIRE].

[4] G. Senjanović, Spontaneous Breakdown of Parity in a Class of Gauge Theories, Nucl. Phys. B 153 (1979) 334 [INSPIRE].

[5] R.N. Mohapatra and G. Senjanović, Neutrino Mass and Spontaneous Parity Violation, Phys. Rev. Lett. 44 (1980) 912 [INSPIRE].

[6] R.N. Mohapatra and G. Senjanović, Neutrino Masses and Mixings in Gauge Models with Spontaneous Parity Violation, Phys. Rev. D 23 (1981) 165 [InSPIRE].

[7] V. Tello, M. Nemevšek, F. Nesti, G. Senjanović and F. Vissani, Left-Right Symmetry: from LHC to Neutrinoless Double Beta Decay, Phys. Rev. Lett. 106 (2011) 151801 [arXiv: 1011.3522] [INSPIRE].

[8] ATLAS collaboration, Search for high-mass diboson resonances with boson-tagged jets in proton-proton collisions at $\sqrt{s}=8 \mathrm{TeV}$ with the ATLAS detector, arXiv:1506.00962 [INSPIRE].

[9] CMS collaboration, Search for heavy neutrinos and $\mathrm{W}$ bosons with right-handed couplings in proton-proton collisions at $\sqrt{s}=8 \mathrm{TeV}$, Eur. Phys. J. C 74 (2014) 3149 [arXiv:1407.3683] [INSPIRE].

[10] CMS collaboration, Search for massive resonances in dijet systems containing jets tagged as $W$ or $Z$ boson decays in pp collisions at $\sqrt{s}=8 \mathrm{TeV}$, JHEP 08 (2014) 173 [arXiv: 1405.1994] [INSPIRE].

[11] CMS collaboration, Search for massive resonances decaying into pairs of boosted bosons in semi-leptonic final states at $\sqrt{s}=8 \mathrm{TeV}$, JHEP 08 (2014) 174 [arXiv:1405.3447] [INSPIRE].

[12] E. Majorana, Theory of the Symmetry of Electrons and Positrons, Nuovo Cim. 14 (1937) 171 [INSPIRE].

[13] GERDA collaboration, M. Agostini et al., Results on Neutrinoless Double- $\beta$ Decay of ${ }^{76}$ Ge from Phase I of the GERDA Experiment, Phys. Rev. Lett. 111 (2013) 122503 [arXiv:1307.4720] [INSPIRE]. 
[14] GERDA collaboration, A.A. Smolnikov, Status of the GERDA experiment aimed to search for neutrinoless double beta decay of ${ }^{76} \mathrm{Ge}$, to appear in proceedings of the 14th International School on Particles and Cosmology, Baksan Valley, Russia, April 16-21 2007 [arXiv:0812.4194] [INSPIRE].

[15] KamLAND-Zen collaboration, A. Gando et al., Limit on Neutrinoless $\beta \beta$ Decay of ${ }^{136} X e$ from the First Phase of KamLAND-Zen and Comparison with the Positive Claim in ${ }^{76} \mathrm{Ge}$, Phys. Rev. Lett. 110 (2013) 062502 [arXiv:1211.3863] [INSPIRE].

[16] EXO collaboration, M. Auger et al., Search for Neutrinoless Double-Beta Decay in ${ }^{136}$ Xe with EXO-200, Phys. Rev. Lett. 109 (2012) 032505 [arXiv:1205.5608] [INSPIRE].

[17] R.N. Mohapatra, New Contributions to Neutrinoless Double beta Decay in Supersymmetric Theories, Phys. Rev. D 34 (1986) 3457 [INSPIRE].

[18] K.S. Babu and R.N. Mohapatra, New vector-scalar contributions to neutrinoless double beta decay and constraints on R-parity violation, Phys. Rev. Lett. 75 (1995) 2276 [hep-ph/9506354] [INSPIRE].

[19] M. Hirsch, H.V. Klapdor-Kleingrothaus and S.G. Kovalenko, New supersymmetric contributions to neutrinoless double beta decay, Phys. Lett. B 352 (1995) 1 [hep-ph/9502315] [INSPIRE].

[20] M. Hirsch, H.V. Klapdor-Kleingrothaus and S.G. Kovalenko, Supersymmetry and neutrinoless double beta decay, Phys. Rev. D 53 (1996) 1329 [hep-ph/9502385] [INSPIRE].

[21] M. Hirsch, H.V. Klapdor-Kleingrothaus and S.G. Kovalenko, New leptoquark mechanism of neutrinoless double beta decay, Phys. Rev. D 54 (1996) 4207 [hep-ph/9603213] [INSPIRE].

[22] G. Racah, On the symmetry of particle and antiparticle, Nuovo Cim. 14 (1937) 322 [INSPIRE].

[23] W.H. Furry, On transition probabilities in double beta-disintegration, Phys. Rev. 56 (1939) 1184 [INSPIRE].

[24] S.P. Das, F.F. Deppisch, O. Kittel and J.W.F. Valle, Heavy Neutrinos and Lepton Flavour Violation in Left-Right Symmetric Models at the LHC, Phys. Rev. D 86 (2012) 055006 [arXiv: 1206.0256] [INSPIRE].

[25] J. Barry and W. Rodejohann, Lepton number and flavour violation in TeV-scale left-right symmetric theories with large left-right mixing, JHEP 09 (2013) 153 [arXiv:1303.6324] [INSPIRE].

[26] P.S. Bhupal Dev, C.-H. Lee and R.N. Mohapatra, Leptogenesis Constraints on the Mass of Right-handed Gauge Bosons, Phys. Rev. D 90 (2014) 095012 [arXiv:1408.2820] [INSPIRE].

[27] F.F. Deppisch, T.E. Gonzalo, S. Patra, N. Sahu and U. Sarkar, Double beta decay, lepton flavor violation and collider signatures of left-right symmetric models with spontaneous D-parity breaking, Phys. Rev. D 91 (2015) 015018 [arXiv:1410.6427] [INSPIRE].

[28] J. Chakrabortty, H.Z. Devi, S. Goswami and S. Patra, Neutrinoless double- $\beta$ decay in TeV scale Left-Right symmetric models, JHEP 08 (2012) 008 [arXiv: 1204.2527] [INSPIRE].

[29] R.L. Awasthi, M.K. Parida and S. Patra, Neutrino masses, dominant neutrinoless double beta decay and observable lepton flavor violation in left-right models and $\mathrm{SO}(10)$ grand unification with low mass $W_{R}, Z_{R}$ bosons, JHEP 08 (2013) 122 [arXiv:1302.0672] [INSPIRE]. 
[30] S.-F. Ge and W. Rodejohann, JUNO and Neutrinoless Double Beta Decay, arXiv: 1507.05514 [INSPIRE].

[31] S.-F. Ge, NuPro: A Simulation Package for Neutrino Physics, http://nupro.hepforge.org/.

[32] S. Dell'Oro, S. Marcocci, M. Viel and F. Vissani, The contribution of light Majorana neutrinos to neutrinoless double beta decay and cosmology, arXiv:1505.02722 [INSPIRE].

[33] D.V. Forero, M. Tortola and J.W.F. Valle, Neutrino oscillations refitted, Phys. Rev. D 90 (2014) 093006 [arXiv: 1405.7540] [INSPIRE].

[34] KATRIN collaboration, A. Osipowicz et al., KATRIN: A Next generation tritium beta decay experiment with sub-eV sensitivity for the electron neutrino mass. Letter of intent, hep-ex/0109033 [INSPIRE].

[35] Planck collaboration, P.A.R. Ade et al., Planck 2013 results. XVI. Cosmological parameters, Astron. Astrophys. 571 (2014) A16 [arXiv:1303.5076] [InSPIRE].

[36] P.S. Bhupal Dev, S. Goswami, M. Mitra and W. Rodejohann, Constraining Neutrino Mass from Neutrinoless Double Beta Decay, Phys. Rev. D 88 (2013) 091301 [arXiv:1305.0056] [INSPIRE].

[37] A. Meroni, S.T. Petcov and F. Simkovic, Multiple CP Non-conserving Mechanisms of $\beta \beta$-Decay and Nuclei with Largely Different Nuclear Matrix Elements, JHEP 02 (2013) 025 [arXiv: 1212.1331] [INSPIRE].

[38] A. Dueck, W. Rodejohann and K. Zuber, Neutrinoless Double Beta Decay, the Inverted Hierarchy and Precision Determination of $\theta_{12}$, Phys. Rev. D 83 (2011) 113010 [arXiv: 1103.4152] [INSPIRE].

[39] JUNO collaboration, F. An et al., Neutrino Physics with JUNO, arXiv:1507.05613 [INSPIRE].

[40] H.V. Klapdor-Kleingrothaus et al., Latest results from the Heidelberg-Moscow double beta decay experiment, Eur. Phys. J. A 12 (2001) 147 [hep-ph/0103062] [INSPIRE].

[41] IGEX collaboration, C.E. Aalseth et al., The IGEX ${ }^{76}$ Ge neutrinoless double beta decay experiment: Prospects for next generation experiments, Phys. Rev. D 65 (2002) 092007 [hep-ex/0202026] [INSPIRE].

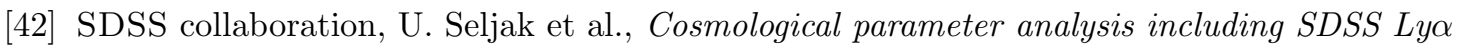
forest and galaxy bias: Constraints on the primordial spectrum of fluctuations, neutrino mass and dark energy, Phys. Rev. D 71 (2005) 103515 [astro-ph/0407372] [INSPIRE].

[43] M. Costanzi, B. Sartoris, M. Viel and S. Borgani, Neutrino constraints: what large-scale structure and CMB data are telling us?, JCAP 10 (2014) 081 [arXiv:1407.8338] [INSPIRE].

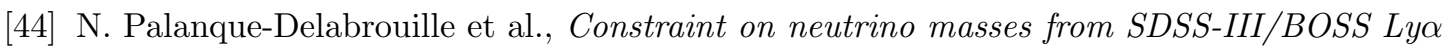
forest and other cosmological probes, JCAP 02 (2015) 045 [arXiv: 1410.7244] [INSPIRE].

[45] A. Faessler, A. Meroni, S.T. Petcov, F. Simkovic and J. Vergados, Uncovering Multiple CP-Nonconserving Mechanisms of $\beta \beta$-Decay, Phys. Rev. D 83 (2011) 113003 [arXiv: 1103.2434] [INSPIRE].

[46] Z.-Z. Xing and Y.-L. Zhou, Geometry of the effective Majorana neutrino mass in the $0 \nu \beta \beta$ decay, Chin. Phys. C 39 (2015) 011001 [arXiv: 1404.7001] [inSPIRE]. 